
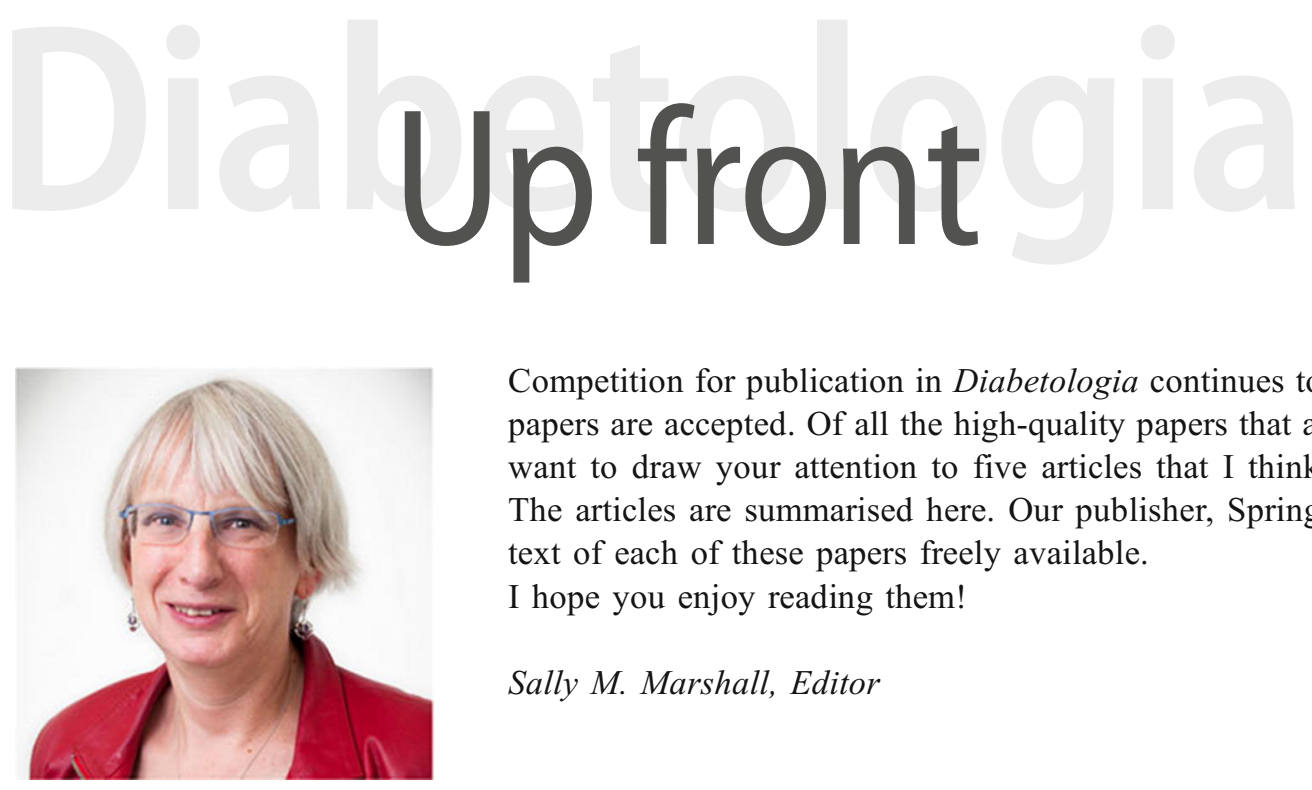

Competition for publication in Diabetologia continues to grow, and less than $20 \%$ of papers are accepted. Of all the high-quality papers that appear in this month's issue I want to draw your attention to five articles that I think are particularly interesting. The articles are summarised here. Our publisher, Springer, has kindly made the full text of each of these papers freely available.

I hope you enjoy reading them!

Sally M. Marshall, Editor

\section{Immunological biomarkers for the development and progression of type 1 diabetes}

Chantal Mathieu, Riitta Lahesmaa, Ezio Bonifacio, Peter Achenbach, Timothy Tree

The target organ of immune destruction in type 1 diabetes is the insulin-producing beta cell. As overall beta cell mass is too small for easy imaging or tissue access, the identification of circulating biomarkers reflecting ongoing immune destruction of beta cells would greatly help the prediction and earlier diagnosis of type 1 diabetes, before onset of hyperglycaemia. In this issue, Mathieu et al (https://doi.org/10.1007/s00125018-4726-8) provide a review of these immunological biomarkers. Some immune biomarkers have reached clinical practice, such as autoantibodies against beta cell antigens, including insulin, GAD, islet antigen- 2 or zinc transporter-8. These autoantibodies are highly predictive for type 1 diabetes risk, both in first-degree family members of people with type 1 diabetes and in the general population. Novel immune biomarkers are emerging, in particular, profiles of circulating $\mathrm{T}$ lymphocyte subsets and their cytokine-producing activity. High hopes have been placed on the upcoming biomarkers, such as microRNA profiles and metabolomic, lipidomic and other 'omic' profiles. These will add power to the more established biomarkers for prediction and diagnosis of type 1 diabetes at earlier stages. The authors note that it is likely that personalised biomarker signatures, combining autoantibodies, T cell profiles and other biomarkers, will be required to categorise at-risk patients, which will facilitate personalised prediction, prevention and treatment approaches.

\section{Biomarkers of islet beta cell stress and death in type 1 diabetes}

Emily K. Sims, Carmella Evans-Molina, Sarah A. Tersey, Decio L. Eizirik, and Raghavendra G. Mirmira

Recent studies have highlighted the heterogenous nature of type 1 diabetes and have suggested that, in some cases, beta cell stress and dysfunction may contribute to and exacerbate autoimmune-mediated beta cell destruction. Robust biomarkers of beta cell stress and death are needed to reflect the pathological contributions of beta cells to this process and to monitor the effect of therapeutic agents that target beta cells in type 1 diabetes prevention or treatment efforts. In this issue, Sims et al (https://doi.org/10.1007/s00125-018-4712-1) provide a review summarising the current beta cell biomarkers, including circulating prohormones, RNA species, extracellular vesicles and differentially methylated DNA species. They also discuss ongoing challenges, such as gaining an understanding of how beta cell health changes as disease develops over time. The authors conclude that a wellvalidated toolkit of biomarkers of beta cell health will allow for a more personalised approach to type 1 diabetes prevention and care.

(t) The figure from this review is available as a downloadable slide. 
Associations of maternal type 1 diabetes with childhood adiposity and metabolic health in the offspring: a prospective cohort study

Anitha Pitchika, Manja Jolink, Christiane Winkler, Sandra Hummel, Nadine Hummel, Jan Krumsiek, Gabi Kastenmüller, Jennifer Raab, Olga Kordonouri, AnetteGabriele Ziegler, Andreas Beyerlein

Children exposed to increased glucose levels in utero may carry additional risks for being overweight and having impaired metabolic health. However, there is only scant evidence from previous studies to support worsening of metabolic health in children of mothers with type 1 diabetes, and potential pathways have not been investigated in detail. In this issue, Pitchika et al (https://doi.org/10.1007/s00125-018-4688-x) report findings from three large studies that examined nearly 2800 children with a first-degree relative with type 1 diabetes. The authors found that children of mothers with type 1 diabetes had a higher risk for being overweight and had increased insulin resistance during childhood and adolescence compared with children of mothers without diabetes. Higher birthweight may partially contribute to this association, but changes in the offspring's metabolome are unlikely to be part of the causal pathway. These findings indicate that children exposed to maternal type 1 diabetes may need closer attention to combat overweight and metabolic risk in later life.

Toll-like receptor 9 negatively regulates pancreatic islet beta cell growth and function in a mouse model of type 1 diabetes

Mengju Liu, Jian Peng, Ningwen Tai, James A. Pearson, Changyun Hu, Junhua Guo, Lin Hou, Hongyu Zhao, F. Susan Wong, Li Wen

Toll-like receptors (TLRs) are known for their role in innate immunity, but information is sparse on their role in tissue development. In this issue, Liu, Peng et al (https://doi.org/ 10.1007/s00125-018-4705-0) demonstrate that TLR9 regulates islet beta cell growth and function in a mouse model of diabetes. They report that TLR9 deficiency results in enhanced beta cell growth, leading to improved glucose tolerance, insulin sensitivity and first-phase insulin secretory response. This effect is mediated, in part, by upregulation of CD140a (also known as platelet-derived growth factor receptor- $\alpha$ ). This novel finding identifies TLR9 as a potential target for the prevention and/or treatment of diabetes.

Innate immune activity as a predictor of persistent insulin secretion and association with responsiveness to CTLA4Ig treatment in recent-onset type 1 diabetes

Susanne M. Cabrera, Samuel Engle, Mary Kaldunski, Shuang Jia, Rhonda Geoffrey, Pippa Simpson, Aniko Szabo, Cate Speake, Carla J. Greenbaum, Type 1 Diabetes TrialNet CTLA4-Ig (Abatacept) Study Group, Yi-Guang Chen, Martin J. Hessner

Recent studies have drawn attention to the phenotypic heterogeneity that exists among individuals with type 1 diabetes. In trials of disease-modifying immunotherapy conducted at clinical onset, heterogeneity in the rate of disease progression poses challenges in detecting the effect of treatment on preservation of stimulated C-peptide. In this issue, Cabrera et al (https://doi.org/10.1007/s00125-018-4708-x) investigated whether discrete subtypes of type 1 diabetes exist, based on immunoregulatory profiles at clinical onset. They report that levels of innate inflammation at clinical onset were very heterogeneous among newly diagnosed individuals. Importantly, the post-onset duration of persistent insulin secretion was negatively related to baseline inflammation and positively associated with baseline abundance of circulating activated regulatory $\mathrm{T}$ cells. Furthermore, in an ancillary analysis of TrialNet CTLA4-Ig trial (TN-09) participants, the therapeutic response to CTLA4-Ig was associated with higher levels of baseline inflammation. These findings suggest that measures that predict the post-onset disease course and the response to therapeutic intervention could enable individual stratification that will lead to the development of individualised therapies.

All text supplied by the authors. 